EPJ Web of Conferences 114,02012 (2016)

DOI: $10.1051 /$ epjconf/201611402012

C Owned by the authors, published by EDP Sciences, 2016

\title{
Finite volume - space-time discontinuous Galerkin method for the solution of compressible turbulent flow
}

Jan Česenek ${ }^{1, a}$

${ }^{1}$ Aerospace Research and Test Establishment, Beranových 130, 19905 Praha - Letňany, Czech Republic

\begin{abstract}
In this article we deal with numerical simulation of the non-stationary compressible turbulent flow. Compressible turbulent flow is described by the Reynolds-Averaged Navier-Stokes (RANS) equations. This RANS system is equipped with two-equation k-omega turbulence model. These two systems of equations are solved separately. Discretization of the RANS system is carried out by the space-time discontinuous Galerkin method which is based on piecewise polynomial discontinuous approximation of the sought solution in space and in time. Discretization of the two-equation k-omega turbulence model is carried out by the implicit finite volume method, which is based on piecewise constant approximation of the sought solution. We present some numerical experiments to demonstrate the applicability of the method using own-developed code.
\end{abstract}

\section{Introduction}

During the last decade the space-time discontinuous Galerkin finite element method (ST-DG), which is based on piecewise polynomial discontinuous approximations of the sought solution, became very popular in the field of numerical simulation of the fluid flow. This method of higher order was successfully used for simulation of the NavierStokes equations ([2],[4],[5]). Unfortunately this method became unstable, when it was used for the whole system of the RANS equations with $k-\omega$ equations. Thus we solve this two systems separately. We use the ST-DG for the discretization of the RANS system of equations and the finite volume method for the equations of the $k-\omega$ turbulence model. As numerical experiments show this is good approach for the solution of this problem.

\section{Formulation of the $k-\omega$ turbulence model}

We consider compressible turbulent flow in a bounded domain $\Omega \subset R^{d}, d=2,3$. We assume that the boundary of $\Omega$ consists of three disjoint parts: $\partial \Omega=\Gamma_{I} \cup \Gamma_{O} \cup \Gamma_{W}$, where $\Gamma_{I}$ is the inlet, $\Gamma_{O}$ is the outlet and $\Gamma_{W}$ is the impermeable wall.

The system of the RANS equations can be written in the following form

$$
\frac{\partial \mathbf{w}}{\partial t}+\sum_{s=1}^{d} \frac{\partial \mathbf{f}_{s}(\mathbf{w})}{\partial x_{s}}=\sum_{s=1}^{d} \frac{\partial \mathbf{R}_{s}(\mathbf{w}, \nabla \mathbf{w})}{\partial x_{s}}
$$

where for $s=1, \ldots, d$ we have

$$
\mathbf{w}=\left(w_{1}, \ldots, w_{d+2}\right)^{T}=\left(\rho, \rho v_{1}, \ldots, \rho v_{d}, E\right)^{T} \in R^{d+2},
$$

\footnotetext{
a e-mail: jan.cessa@seznam.cz, cesenek@vzlu.cz
}

$$
\begin{aligned}
\mathbf{f}_{s}(\mathbf{w})= & \left(f_{s, 1}, \ldots, f_{s, d+2}\right)^{T} \\
= & \left(\rho v_{s}, \rho v_{1} v_{s}+\delta_{1 s}\left(p+\frac{2}{3} \rho k\right), \ldots,\right. \\
& \left.\rho v_{d} v_{s}+\delta_{d s}\left(p+\frac{2}{3} \rho k\right),\left(E+p+\frac{2}{3} \rho k\right) v_{s}\right)^{T}, \\
& \mathbf{R}_{s}(\mathbf{w}, \nabla \mathbf{w})=\left(R_{s, 1}, \ldots, R_{s, d+2}\right)^{T} \\
= & \left(0, \tau_{s 1}^{V}, \ldots, \tau_{s d}^{V}, \sum_{r=1}^{d} \tau_{s r}^{V} v_{r}+\left(\frac{c_{p} \mu_{L}}{P r}+\frac{c_{p} \mu_{T}}{P r_{T}}\right) \frac{\partial \theta}{\partial x_{s}}\right)^{T},
\end{aligned}
$$

where

$$
\begin{aligned}
\tau_{s r}^{V} & =-\frac{2}{3}\left(\mu_{L}+\mu_{T}\right) \operatorname{div} \boldsymbol{v} \delta_{s r}+2\left(\mu_{L}+\mu_{T}\right) d_{s r}(\boldsymbol{v}), \\
d_{s r}(\boldsymbol{v}) & =\frac{1}{2}\left(\frac{\partial v_{s}}{\partial x_{r}}+\frac{\partial v_{r}}{\partial x_{s}}\right) .
\end{aligned}
$$

We use the following notation: $\boldsymbol{v}=\left(v_{1}, \ldots, v_{d}\right)$ - velocity, $\rho$ - density, $p$ - pressure, $\theta$ - absolute temperature, $E$ - total energy, $\gamma$ - Poisson adiabatic constant, $\kappa$ - heat conduction coefficient, $c_{v}$ - specific heat at constant volume, $c_{p}$ - specific heat at constant pressure, where $c_{p}=\gamma c_{v}, \operatorname{Pr}$ is the laminar Prandtl number, which can be express in the form $\operatorname{Pr}=\frac{c_{p} \mu_{L}}{K}, \operatorname{Pr}_{T}$ is the turbulent Prandtl constant number, $\mu_{T}$ is the eddy-viscosity coefficient, $\mu_{L}$ is the dynamic viscosity coefficient dependent on temperature via Sutherland's formula. The above system is completed by the thermodynamical relations

$$
p=(\gamma-1)\left(E-\frac{1}{2} \rho|\boldsymbol{v}|^{2}-\rho k\right), \quad \theta=\frac{1}{c_{v}}\left(\frac{E}{\rho}-\frac{1}{2}|\boldsymbol{v}|^{2}-k\right)
$$

and is equipped with the initial condition

$$
\mathbf{w}(x, 0)=\mathbf{w}^{0}(x), \quad x \in \Omega
$$

and the following boundary conditions

a) $\left.\rho\right|_{\Gamma_{I}}=\rho_{D}$, 
b) $\left.\boldsymbol{v}\right|_{\Gamma_{I}}=\boldsymbol{v}_{D}=\left(v_{D 1}, \ldots, v_{D d}\right)^{T}$,

c) $\sum_{s, r=1}^{d} \tau_{s r}^{V} n_{s} v_{r}+\left(\frac{c_{p} \mu_{L}}{P r}+\frac{c_{p} \mu_{T}}{P r_{T}}\right) \frac{\partial \theta}{\partial n}=0 \quad$ on $\Gamma_{I}$,

a) $\left.\boldsymbol{v}\right|_{\Gamma_{W}}=\mathbf{0}$,

b) $\left.\frac{\partial \theta}{\partial n}\right|_{\Gamma_{W}}=\mathbf{0}$

a) $\sum_{s=1}^{2} \tau_{s r}^{V} n_{s}=0, r=1,2, \quad$ on $\Gamma_{O}, \quad$ b) $\left.\frac{\partial \theta}{\partial n}\right|_{\Gamma_{O}}=\mathbf{0}$

with given data $\mathbf{w}^{0}, \rho_{D}, \boldsymbol{v}_{D}$. It is possible to show that $\mathbf{f}_{s}(\alpha \mathbf{w})=$ $\alpha \mathbf{f}_{s}(\mathbf{w})$ for $\alpha>0$. This property implies that

$$
\mathbf{f}_{s}(\mathbf{w})=\mathbf{A}_{s}(\mathbf{w}) \mathbf{w}, \quad s=1, \ldots, d,
$$

where

$$
\mathbf{A}_{s}(\mathbf{w})=\frac{D \mathbf{f}_{s}(\mathbf{w})}{D \mathbf{w}}, \quad s=1, \ldots, d,
$$

are the Jacobi matrices of the mappings $\mathbf{f}_{s}$. The viscous terms $\mathbf{R}_{s}(\mathbf{w}, \nabla \mathbf{w})$ can be expressed in the form

$$
\mathbf{R}_{s}(\mathbf{w}, \nabla \mathbf{w})=\sum_{k=1}^{d} \mathbf{K}_{s, k}(\mathbf{w}) \frac{\partial \mathbf{w}}{\partial x_{k}}, \quad s=1,2,
$$

where $\mathbf{K}_{s, k}(\mathbf{w}) \in \boldsymbol{R}^{(d+2) \times(d+2)}$ are matrices depending on $\mathbf{w}$.

The aforesaid system of the RANS equations has to be completed by a turbulence model for $\mu_{T}$ :

$$
\frac{\partial \tilde{\mathbf{w}}}{\partial t}+\sum_{s=1}^{d} \frac{\partial \tilde{\mathbf{f}}_{s}(\tilde{\mathbf{w}})}{\partial x_{s}}=\sum_{s=1}^{d} \frac{\partial \tilde{\mathbf{R}}_{s}(\tilde{\mathbf{w}}, \nabla \tilde{\mathbf{w}})}{\partial x_{s}}+\tilde{\mathbf{s}}(\tilde{\mathbf{w}}),
$$

where for $s=1, \ldots, d$ we have

$$
\begin{aligned}
\tilde{\mathbf{w}}= & \left(\tilde{w}_{1}, \tilde{w}_{2}\right)^{T}=(\rho \omega, \rho k)^{T} \in R^{2}, \\
\tilde{\mathbf{f}}_{s}(\tilde{\mathbf{w}})= & \left(\tilde{f}_{s, 1}, \tilde{f}_{s, 2}\right)^{T}=\left(\rho \omega v_{s}, \rho k v_{s}\right)^{T}, \\
\tilde{\mathbf{R}}_{s}(\tilde{\mathbf{w}}, \nabla \tilde{\mathbf{w}})= & \left(R_{s, 1}, R_{s, 2}\right)^{T}= \\
& \left(\left(\mu_{L}+\sigma_{\omega} \mu_{T}\right) \frac{\partial \omega}{\partial x_{s}},\left(\mu_{L}+\sigma_{k} \mu_{T}\right) \frac{\partial k}{\partial x_{s}}\right)^{T}, \\
\tilde{\mathbf{s}}(\tilde{\mathbf{w}})= & \left(P_{\omega}-\beta \rho \omega^{2}+C_{D}, P_{k}-\beta^{*} \rho \omega k\right)^{T} .
\end{aligned}
$$

Here $\omega$ is the turbulence dissipation, $k$ is the turbulence kinetic energy and $\mu_{T}=\frac{\rho k}{\omega}$ is the eddy viscosity. We can write the production terms as

$$
\begin{aligned}
& P_{k}=\sum_{s=1}^{d} \sum_{r=1}^{d} \tau_{s r}^{T} \frac{\partial v_{s}}{\partial x_{r}}, \\
& P_{\omega}=\alpha_{\omega} \frac{\omega P_{k}}{k},
\end{aligned}
$$

where

$$
\begin{aligned}
\tau_{s r}^{T} & =-\frac{2}{3} \mu_{T} \operatorname{div} \boldsymbol{v} \delta_{s r}-\frac{2}{3} \rho k \delta_{s r}+2 \mu_{T} d_{s r}(\boldsymbol{v}), \\
d_{s r}(\boldsymbol{v}) & =\frac{1}{2}\left(\frac{\partial v_{s}}{\partial x_{r}}+\frac{\partial v_{r}}{\partial x_{s}}\right) .
\end{aligned}
$$

The cross-diffusion term $C_{D}$ is defined as

$$
C_{D}=\sigma_{D} \frac{\rho}{\omega} \max \left\{\sum_{s=1}^{d} \frac{\partial k}{\partial x_{s}} \frac{\partial \omega}{\partial x_{s}}, 0\right\} .
$$

The coefficients $\beta, \beta^{*}, \sigma_{k}, \sigma_{\omega}, \sigma_{D}, \alpha_{\omega}$ are chosen by [9]:

$$
\begin{aligned}
& \beta^{*}=0.09, \quad \beta=\frac{5}{6} \beta^{*}, \quad \sigma_{\omega}=0.5, \quad \sigma_{k}=\frac{2}{3}, \\
& \kappa=0.41, \quad \sigma_{D}=0.5, \quad \alpha_{\omega}=\frac{\beta}{\beta^{*}}-\sigma_{\omega} \frac{\kappa^{2}}{\sqrt{\beta^{*}}} .
\end{aligned}
$$

This system is also equipped with the initial condition

$$
\tilde{\mathbf{w}}(x, 0)=\tilde{\mathbf{w}}^{0}(x), \quad x \in \Omega
$$

and the following boundary conditions
a) $\left.\omega\right|_{\Gamma_{I}}=\omega_{D}$,
b) $\left.k\right|_{\Gamma_{I}}=k_{D}$,
a) $\left.\omega\right|_{\Gamma_{W}}=\omega_{\text {wall }}$,
b) $\left.k\right|_{\Gamma_{W}}=0$,
a) $\left.\frac{\partial \omega}{\partial n}\right|_{\Gamma_{O}}=\mathbf{0}$
b) $\left.\frac{\partial k}{\partial n}\right|_{\Gamma_{O}}=\mathbf{0}$,

with given data $\tilde{\mathbf{w}}^{0}, \omega_{D}, k_{D}, \omega_{\text {wall }}$.

\section{Space-time discretization of the RANS equations}

\subsection{Space discretization}

By $\Omega_{h}$ we denote a polygonal $(\mathrm{d}=2)$ or polyhedral $(\mathrm{d}=3)$ approximation of the domain $\Omega$. Let $\mathcal{T}_{h}$ be a partition of the domain $\Omega_{h}$ into finite number of closed elements with mutually disjoint interiors such that $\overline{\Omega_{h}}=\bigcup_{K \in \mathcal{T}_{h}} K$. In 2D problems, we usually choose $K \in \mathcal{T}_{h}$ as triangles or quadrilaterals. In $3 \mathrm{D}, K \in \mathcal{T}_{h}$ can be, e.g., tetrahedrons, prisms or hexahedrons. By $\mathcal{F}_{h}$ we denote the system of all faces of all elements $K \in \mathcal{T}_{h}$. Further, we introduce the set of boundary faces $\mathcal{F}_{h}^{B}=\left\{\Gamma \in \mathcal{F}_{h} ; \Gamma \subset \partial \Omega_{h}(t)\right\}$ and the set of inner faces $\mathcal{F}_{h}^{I}=\mathcal{F}_{h} \backslash \mathcal{F}_{h}^{B}$. Each $\Gamma \in \mathcal{F}_{h}$ is associated with a unit normal vector $\boldsymbol{n}_{\Gamma}$. For $\Gamma \in \mathcal{F}_{h}^{B}$ the normal $\boldsymbol{n}_{\Gamma}$ has the same orientation as the outer normal to $\partial \Omega_{h}$. For each $\Gamma \in \mathcal{F}_{h}^{I}$ there exist two neighbouring elements $K_{\Gamma}^{L}, K_{\Gamma}^{R} \in \mathcal{T}_{h}$ such that $\Gamma \subset \partial K_{\Gamma}^{L} \cap \partial K_{\Gamma}^{R}$. We use the convention that $K_{\Gamma}^{R}$ lies in the direction of $\boldsymbol{n}_{\Gamma}$ and $K_{\Gamma}^{L}$ lies in the opposite direction to $\boldsymbol{n}_{\Gamma}$. If $\Gamma \in \mathcal{F}_{h}^{B}$, then the element adjacent to $\Gamma$ will be denoted by $K_{\Gamma}^{L}$.

Because of the space discretization we define the space of piecewise polynomial functions

$$
\mathbf{S}_{h}^{p}=\left(S_{h}^{p}\right)^{d+2}, \quad S_{h}^{p}=\left\{v ;\left.v\right|_{K} \in P^{p}(K), \forall K \in \mathcal{T}_{h}\right\},
$$

where $p>0$ is an integer and $P^{p}(K)$ denotes the space of all polynomials on $K$ of degree $\leq p$. A function $\Phi \in \mathbf{S}_{h}^{p}$ is, in general, discontinuous on interfaces $\Gamma \in \mathcal{F}_{h}^{I}$. By $\Phi_{\Gamma}^{L}$ and $\Phi_{\Gamma}^{R}$ we denote the values of $\Phi$ on $\Gamma$ considered from the interior and the exterior of $K_{\Gamma}^{L}$, respectively, and set

$$
\begin{aligned}
& \langle\Phi\rangle_{\Gamma}=\frac{1}{2}\left(\Phi_{\Gamma}^{L}+\Phi_{\Gamma}^{R}\right), \\
& {[\Phi]_{\Gamma}=\Phi_{\Gamma}^{L}-\Phi_{\Gamma}^{R},}
\end{aligned}
$$

which denotes average and jump of $\Phi$ on $\Gamma$.

The discrete problem is derived in the following way: For arbitrary $t \in[0, T]$ we can multiply the system (1) by a test function $\Phi_{h} \in \mathbf{S}_{h}^{p}$, integrate over $K \in \mathcal{T}_{h}$, apply Green's 
theorem, sum over all elements $K \in \mathcal{T}_{h}$, use the concept of the numerical flux and introduce suitable terms mutually vanishing for a regular exact solution. Moreover, we carry out a suitable partial linearization of nonlinear terms.

In order to evaluate the integrals over $\Gamma \in \mathcal{F}_{h}$ in inviscid term we use the approximation

$$
\mathbf{H}\left(\mathbf{w}_{\Gamma}^{L}, \mathbf{w}_{\Gamma}^{R}, \boldsymbol{n}_{\Gamma}\right) \approx \sum_{s=1}^{d} \mathbf{f}_{s}(\mathbf{w})\left(\boldsymbol{n}_{\Gamma}\right)_{s},
$$

where $\mathbf{H}$ is a numerical flux. For the construction of the numerical flux we use the properties (3) of $\mathbf{f}_{s}$. Let us define the matrix

$$
\mathbf{P}(\mathbf{w}, \boldsymbol{n}):=\sum_{s=1}^{d} \mathbf{A}_{s}(\mathbf{w}) n_{s}
$$

where $\boldsymbol{n}=\left(n_{1}, \ldots, n_{d}\right), n_{1}^{2}+\ldots+n_{d}^{2}=1$. Then we have

$$
\mathbf{P}(\mathbf{w}, \boldsymbol{n}) \mathbf{w}:=\sum_{s=1}^{d} \mathbf{f}_{s}(\mathbf{w}) n_{s} .
$$

It is possible to show that the matrix $\mathbf{P}$ is diagonalizable. It means that there exists a nonsingular matrix $\mathbf{T}=\mathbf{T}(\mathbf{w}, \boldsymbol{n})$ and a diagonal matrix $\Lambda=\Lambda(\mathbf{w}, \boldsymbol{n})$ such that

$$
\mathbf{P}=\mathbf{T} \Lambda \mathbf{T}^{-1}, \quad \Lambda=\operatorname{diag}\left(\lambda_{1}, \ldots, \lambda_{d+2}\right),
$$

where $\lambda_{i}=\lambda_{i}(\mathbf{w}, \boldsymbol{n})$ are eigenvalues of the matrix $\mathbf{P}$. Then we can define the "positive" and "negative" parts of the matrix $\mathbf{P}$ by

$$
\mathbf{P}^{ \pm}=\mathbf{T} \Lambda^{ \pm} \mathbf{T}^{-1}, \quad \Lambda^{ \pm}=\operatorname{diag}\left(\lambda_{1}^{ \pm}, \ldots, \lambda_{d+2}^{ \pm}\right),
$$

where $\lambda^{+}=\max (\lambda, 0), \lambda^{-}=\min (\lambda, 0)$. Using this concept, we introduce the so-called Vijayasundaram numerical flux

$$
\mathbf{H}\left(\mathbf{w}^{L}, \mathbf{w}^{R}, \boldsymbol{n}\right)=\mathbf{P}^{+}\left(\frac{\mathbf{w}^{L}+\mathbf{w}^{R}}{2}, \boldsymbol{n}\right) \mathbf{w}^{L}+\mathbf{P}^{-}\left(\frac{\mathbf{w}^{L}+\mathbf{w}^{R}}{2}, \boldsymbol{n}\right) \mathbf{w}^{R} .
$$

This numerical flux has suitable form for a linearization. Now we can define inviscid form in the following way:

$$
\begin{aligned}
& b_{h}\left(\overline{\mathbf{w}}_{h}, \mathbf{w}_{h}, \Phi_{h}\right):= \\
& -\sum_{K \in \mathcal{T}_{h}} \int_{K} \sum_{s=1}^{d} \mathbf{A}_{s}\left(\overline{\mathbf{w}}_{h}\right) \mathbf{w}_{h} \cdot \frac{\partial \Phi_{h}}{\partial x_{s}} \mathrm{~d} \boldsymbol{x} \\
& +\sum_{\Gamma \in \mathcal{F}_{h}^{I}} \int_{\Gamma}\left(\mathbf{P}^{+}\left(\left\langle\overline{\mathbf{w}}_{h}\right\rangle, \boldsymbol{n}_{\Gamma}\right) \mathbf{w}_{h}^{L}+\mathbf{P}^{-}\left(\left\langle\overline{\mathbf{w}}_{h}\right\rangle, \boldsymbol{n}_{\Gamma}\right) \mathbf{w}_{h}^{R}\right) \cdot\left[\Phi_{h}\right] \mathrm{d} S \\
& +\sum_{\Gamma \in \mathcal{F}_{h}^{B}} \int_{\Gamma}\left(\mathbf{P}^{+}\left(\left\langle\overline{\mathbf{w}}_{h}\right\rangle, \boldsymbol{n}_{\Gamma}\right) \mathbf{w}_{h}^{L}+\mathbf{P}^{-}\left(\left\langle\overline{\mathbf{w}}_{h}\right\rangle, \boldsymbol{n}_{\Gamma}\right) \overline{\mathbf{w}}_{h}^{R}\right) \cdot \Phi_{h} \mathrm{~d} S
\end{aligned}
$$

The boundary state $\overline{\mathbf{w}}_{h}^{R}$ is evaluated with the aid of the local linearized Riemann problem described in [8].

For the discretization of the viscous terms we use the property (4) and get the viscous form

$$
\begin{aligned}
& a_{h}\left(\overline{\mathbf{w}}_{h}, \mathbf{w}_{h}, \Phi_{h}\right):= \\
& +\sum_{K \in \mathcal{T}_{h}} \int_{K} \sum_{s=1}^{d} \sum_{k=1}^{d} \mathbf{K}_{s, k}\left(\overline{\mathbf{w}}_{h}\right) \frac{\partial \mathbf{w}_{h}}{\partial x_{k}} \cdot \frac{\partial \Phi_{h}}{\partial x_{s}} \mathrm{~d} \boldsymbol{x}
\end{aligned}
$$

$$
\begin{aligned}
& -\sum_{\Gamma \in \mathcal{F}_{h}^{I}} \int_{\Gamma} \sum_{s=1}^{d}\left\langle\sum_{k=1}^{d} \mathbf{K}_{s, k}\left(\overline{\mathbf{w}}_{h}\right) \frac{\partial \mathbf{w}_{h}}{\partial x_{k}}\right\rangle\left(\boldsymbol{n}_{\Gamma}\right)_{s} \cdot\left[\Phi_{h}\right] \mathrm{d} S \\
& -\sum_{\Gamma \in \mathcal{F}_{h}^{B}} \int_{\Gamma} \sum_{s=1}^{d} \sum_{k=1}^{d} \mathbf{K}_{s, k}\left(\overline{\mathbf{w}}_{h}\right) \frac{\partial \mathbf{w}_{h}}{\partial x_{k}}\left(\boldsymbol{n}_{\Gamma}\right)_{s} \cdot \Phi_{h} \mathrm{~d} S \\
& -\Theta \sum_{\Gamma \in \mathcal{F}_{h}^{I}} \int_{\Gamma} \sum_{s=1}^{d}\left\langle\sum_{k=1}^{d} \mathbf{K}_{k, s}^{T}\left(\overline{\mathbf{w}}_{h}\right) \frac{\partial \Phi_{h}}{\partial x_{k}}\right\rangle\left(\boldsymbol{n}_{\Gamma}\right)_{s} \cdot\left[\mathbf{w}_{h}\right] \mathrm{d} S \\
& -\Theta \sum_{\Gamma \in \mathcal{F}_{h}^{B}} \int_{\Gamma} \sum_{s=1}^{d} \sum_{k=1}^{d} \mathbf{K}_{k, s}^{T}\left(\overline{\mathbf{w}}_{h}\right) \frac{\partial \Phi_{h}}{\partial x_{k}}\left(\boldsymbol{n}_{\Gamma}\right)_{s} \cdot \mathbf{w}_{h} \mathrm{~d} S .
\end{aligned}
$$

We set $\Theta=1$ or $\Theta=0$ or $\Theta=-1$ and get the so-called symmetric version (SIPG) or incomplete version (IIPG) or nonsymetric version (NIPG), respectively, of the discretization of viscous terms.

Further, we define the interior and boundary penalty form $J_{h}^{\sigma}$ and the right-hand side form $l_{h}$ in the following way:

$$
\begin{aligned}
& J_{h}^{\sigma}\left(\mathbf{w}_{h}, \Phi_{h}\right):=\sum_{\Gamma \in \mathcal{F}_{h}^{I}} \int_{\Gamma} \sigma\left[\mathbf{w}_{h}\right] \cdot\left[\Phi_{h}\right] \mathrm{d} S \\
& +\sum_{\Gamma \in \mathcal{F}_{h}^{B}} \int_{\Gamma} \sigma \mathbf{w}_{h} \cdot \Phi_{h} \mathrm{~d} S, \\
& l_{h}\left(\overline{\mathbf{w}}_{h}, \Phi_{h}\right):=\sum_{\Gamma \in \mathcal{F}_{h}^{B}} \int_{\Gamma} \sigma \mathbf{w}_{B} \cdot \Phi_{h} \mathrm{~d} S \\
& -\Theta \sum_{\Gamma \in \mathcal{F}_{h}^{B}} \int_{\Gamma} \sum_{s=1}^{d} \sum_{k=1}^{d} \mathbf{K}_{k, s}^{T}\left(\overline{\mathbf{w}}_{h}\right) \frac{\partial \Phi_{h}}{\partial x_{k}}\left(\boldsymbol{n}_{\Gamma}\right)_{s} \cdot \mathbf{w}_{B} \mathrm{~d} S,
\end{aligned}
$$

where $\left.\sigma\right|_{\Gamma}=\frac{C_{W}}{\operatorname{Red} d(\Gamma)}$, where $d(\Gamma)$ is the diameter of $\Gamma \in \mathcal{F}_{h}$, $R e$ is the Reynolds number and $C_{W}>0$ is a suitable sufficiently large constant. The boundary state $\mathbf{w}_{B}$ is defined on the basis of the Dirichlet boundary conditions and extrapolation:

$$
\begin{aligned}
\mathbf{w}_{B}= & \left(\rho_{D}, \rho_{D} v_{D 1}, \ldots,\right. \\
& \left.\rho_{D} v_{D d}, c_{v} \rho_{D} \theta_{\Gamma}^{L}+\frac{1}{2} \rho_{D}\left|\boldsymbol{v}_{D}\right|^{2}+\rho_{D} k_{D}\right) \quad \text { on } \Gamma_{I}, \\
\mathbf{w}_{B}= & \mathbf{w}_{\Gamma}^{L} \quad \text { on } \Gamma_{O}, \\
\mathbf{w}_{B}= & \left(\rho_{\Gamma}^{L}, 0, \ldots, 0, c_{v} \rho_{\Gamma}^{L} \theta_{\Gamma}^{L}+\frac{1}{2} \rho_{\Gamma}^{L}\left|z_{D}\right|^{2}\right) \quad \text { on } \Gamma_{W} .
\end{aligned}
$$

In the vicinity of discontinuities or steep gradients nonphysical oscillations can appear in the approximate solution. In order to overcome this difficulty we employ artificial viscosity form, see [7]. They are based on the discontinuity indicator

$$
g(K):=\frac{1}{d(K)|K|^{3 / 4}} \int_{\partial K}\left[\bar{\rho}_{h}\right]^{2} d S, \quad K \in \mathcal{T}_{h},
$$

where $\left[\bar{\rho}_{h}\right]$ is the jump of the function $\bar{\rho}_{h}$ (= the first component of the vector function $\overline{\mathbf{w}}_{h}$ ) on the boundary $\partial K$, $d(K)$ denotes the diameter of $K$ and $|K|$ denotes the area of the element $K$. Then we define the discrete discontinuity indicator

$$
\begin{array}{ll}
G(K):=0 & \text { if } g(K)<1, \\
G(K):=1 & \text { if } g(K) \geq 1, K \in \mathcal{T}_{h},
\end{array}
$$


and the artificial viscosity forms

$$
\begin{aligned}
& \tilde{\beta}_{h}\left(\overline{\mathbf{w}}_{h}, \mathbf{w}_{h}, \Phi_{h}\right):= \\
& v_{1} \sum_{K \in \mathcal{T}_{h}} d(K) G(K) \int_{K} \nabla \mathbf{w}_{h} \cdot \nabla \Phi_{h} \mathrm{~d} \boldsymbol{x}, \\
& \tilde{J}_{h}\left(\overline{\mathbf{w}}_{h}, \mathbf{w}_{h}, \Phi_{h}\right):= \\
& v_{2} \sum_{\Gamma \in \mathcal{F}_{h}^{I}} \frac{1}{2}\left(G\left(K_{\Gamma}^{L}\right)+G\left(K_{\Gamma}^{R}\right)\right) \int_{\Gamma}\left[\mathbf{w}_{h}\right] \cdot\left[\Phi_{h}\right] \mathrm{d} S,
\end{aligned}
$$

with constants $v_{1}$ and $v_{2}$.

All these forms are linear with respect to $\mathbf{w}_{h}$ and nonlinear with respect to $\overline{\mathbf{w}}_{h}$.

Finally, we set

$$
(\phi, \psi)=\int_{\Omega_{h}} \phi \psi \mathrm{d} \mathbf{x}
$$

\subsection{Full space-time DGM discretization}

Let $0=t_{0}<t_{1}<\ldots<t_{M}=T$ be a partition of the interval $[0, T]$ and denote $I_{m}=\left(t_{m-1}, t_{m}\right), \tau_{m}=t_{m}-t_{m-1}$ for $m=1, \ldots, M$. We define the space $\mathbf{S}_{h, \tau}^{p, q}=\left(S_{h, \tau}^{p, q}\right)^{d+2}$, where

$$
S_{h, \tau}^{p, q}:=\left\{\phi ;\left.\phi\right|_{I_{m}}=\sum_{i=0}^{q} \zeta_{i} \phi_{i}, \text { where } \phi_{i} \in S_{h}^{p}, \zeta_{i} \in P^{q}\left(I_{m}\right)\right\}
$$

with integers $p, q \geq 1$. $P^{q}\left(I_{m}\right)$ denotes the space of all polynomials in $t$ on $I_{m}$ of degree $\leq q$. Moreover for $\Phi \in \mathbf{S}_{h, \tau}^{p, q}$ we introduce the following notation:

$$
\begin{aligned}
& \Phi_{m}^{ \pm}=\Phi\left(t_{m}^{ \pm}\right)=\lim _{t \rightarrow t_{m} \pm} \Phi(t), \\
& \{\Phi\}_{m}=\Phi_{m}^{+}-\Phi_{m}^{-} .
\end{aligned}
$$

Approximate solution $\mathbf{w}_{h \tau}$ of the problem will be sought in the space $\mathbf{S}_{h, \tau}^{p, q}$. Since the functions of this space are in general discontinuous in time, we ensure the connection between $I_{m-1}$ and $I_{m}$ by the penalty term in time

$$
\left(\left\{\mathbf{w}_{h \tau}\right\}_{m-1}, \Phi_{h \tau}\left(t_{m-1}^{+}\right)\right) .
$$

The initial state $\mathbf{w}_{h \tau}$ is included by the $L^{2}\left(\Omega_{h}\right)$-projection of $\mathbf{w}^{0}$ on $\mathbf{S}_{h}^{p}$ :

$$
\left(\mathbf{w}_{h \tau}\left(t_{0}^{+}\right), \Phi_{h \tau}\left(t_{0}^{+}\right)\right)=\left(\mathbf{w}^{0}, \Phi_{h \tau}\left(t_{0}^{+}\right)\right) \quad \forall \Phi_{h \tau} \in \mathbf{S}_{h, \tau}^{p, q} .
$$

Now we introduce a suitable linearization. We can use two possibilities.

1) We put $\overline{\mathbf{w}}_{h \tau}(t):=\mathbf{w}_{h}\left(t_{m-1}^{-}\right)$for $t \in I_{m}$.

2) We prolong the solution from the time interval $I_{m-1}$ to the time interval $I_{m}$.

We say that a function $\mathbf{w}_{h \tau} \in \mathbf{S}_{h, \tau}^{p, q}$ is the approximate solution of the problem (1) obtained by the ST-DG method, if it satisfies the following conditions

$$
\begin{aligned}
& \sum_{m=1}^{M} \int_{I_{m}}\left(\frac{\partial \mathbf{w}_{h \tau}}{\partial t}, \Phi_{h \tau}\right) \mathrm{d} t \\
& +\sum_{m=1}^{M} \int_{I_{m}}\left(a_{h}\left(\overline{\mathbf{w}}_{h \tau}, \mathbf{w}_{h \tau}, \Phi_{h \tau}\right)+b_{h}\left(\overline{\mathbf{w}}_{h \tau}, \mathbf{w}_{h \tau}, \Phi_{h \tau}\right)\right) \mathrm{d} t
\end{aligned}
$$

$$
\begin{aligned}
& +\sum_{m=1}^{M} \int_{I_{m}}\left(\tilde{\beta}_{h}\left(\overline{\mathbf{w}}_{h \tau}, \mathbf{w}_{h \tau}, \Phi_{h \tau}\right)+\tilde{J}_{h}\left(\overline{\mathbf{w}}_{h \tau}, \mathbf{w}_{h \tau}, \Phi_{h \tau}\right)\right) \mathrm{d} t \\
& +\sum_{m=1}^{M} \int_{I_{m}} J_{h}^{\sigma}\left(\mathbf{w}_{h \tau}, \Phi_{h \tau}\right) \mathrm{d} t \\
& +\sum_{m=2}^{M}\left(\left\{\mathbf{w}_{h \tau}\right\}_{m-1}, \Phi_{h \tau}\left(t_{m-1}^{+}\right)\right)+\left(\mathbf{w}_{h \tau}\left(t_{0}^{+}\right), \Phi_{h \tau}\left(t_{0}^{+}\right)\right) \\
& =\sum_{m=1}^{M} \int_{I_{m}} l_{h}\left(\overline{\mathbf{w}}_{h \tau}, \Phi_{h \tau}\right) \mathrm{d} t+\left(\mathbf{w}^{0}, \Phi_{h \tau}\left(t_{0}^{+}\right)\right) \quad \forall \Phi_{h \tau} \in \mathbf{S}_{h, \tau}^{p, q} .
\end{aligned}
$$

\section{Finite volume discretization of the two-equation $k-\omega$ turbulence model}

Similarly as in Section 3.1 let $\mathcal{T}_{h}=\left\{K_{i}\right\}_{i \in I}$ be a partition of the domain $\Omega_{h}$ into finite number of closed elements with mutually disjoint interiors such that $\bar{\Omega}_{h}=\bigcup_{i \in I} K_{i}$, where $I \subset Z^{+}=\{0,1,2,3, \ldots\}$ is a suitable index set. If two elements have a common face, then we call them neighbours and put $\Gamma_{i j}=\Gamma_{j i}=\partial K_{i} \cap \partial K_{j}$. For each $i \in I$ we define the index set $s(i)=\left\{j \in I ; K_{j}\right.$ is a neighbour of $\left.K_{i}\right\}$. The boundary $\partial \Omega_{h}$ is formed by a finite number of sides of elements $K_{i}$ adjacent to $\partial \Omega_{h}$. We denote all these boundary sides by $S_{j}$, where $j \in I_{b} \subset Z^{-}=\{-1,-2,-3, \ldots\}$ and set $\gamma(i)=\left\{j \in I_{b} ; S_{j}\right.$ is a side of $\left.K_{i}\right\}, \Gamma_{i j}=S_{j}$ for $K_{i} \in \mathcal{T}_{h}$ such that $S_{j} \subset \partial K_{i}, j \in I_{b}$. For an element $K_{i}$, not containing any boundary side $S_{j}$, we set $\gamma(i)=\emptyset$. Moreover we define $S(i)=s(i) \cup \gamma(i)$ and $\boldsymbol{n}_{i j}=\left(\left(n_{i j}\right)_{1}, \ldots,\left(n_{i j}\right)_{d}\right)$ as the unit outer normal to $\partial K_{i}$ on the side $\Gamma_{i j}$. We again consider a partition $0=t_{0}<t_{1}<\ldots<t_{M}=T$ of the interval $[0, T]$ and denote $I_{m}=\left(t_{m-1}, t_{m}\right), \tau_{m}=t_{m}-t_{m-1}$ for $m=1, \ldots, M$.

Now we can integrate the system (5) over set $K_{i} \times$ $\left(t_{m-1}, t_{m}\right)$, apply Green's theorem and approximate the integrals with the one-point rule and we get the following implicit finite volume scheme,

$$
\begin{aligned}
& \left|K_{i}\right|\left(\tilde{\mathbf{w}}_{i}^{m}-\tilde{\mathbf{w}}_{i}^{m-1}\right)-\tau_{m}\left|K_{i}\right| \tilde{\mathbf{s}}\left(\tilde{\mathbf{w}}_{i}^{m}\right) \\
+ & \tau_{m} \sum_{j \in S(i)}\left|\Gamma_{i j}\right| \tilde{\mathbf{H}}\left(\tilde{\mathbf{w}}_{i}^{m}, \tilde{\mathbf{w}}_{j}^{m}, \boldsymbol{n}_{i j}\right) \\
- & \tau_{m} \sum_{j \in S(i)}\left|\Gamma_{i j}\right| \sum_{s=1}^{d} \tilde{\mathbf{R}}_{s}\left(\tilde{\mathbf{w}}_{\Gamma_{i j}}^{m}, \nabla \tilde{\mathbf{w}}_{\Gamma_{i j}}^{m}\right)\left(n_{i j}\right)_{s} \\
= & 0 \quad \forall i \in I .
\end{aligned}
$$

Here $\tilde{\mathbf{w}}_{\Gamma_{i j}}^{m}$ resp. $\nabla \tilde{\mathbf{w}}_{\Gamma_{i j}}^{m}$ denote $\tilde{\mathbf{w}}^{m}$ resp. $\nabla \tilde{\mathbf{w}}^{m}$ at the center of the edge $\Gamma_{i j}$ at time instant $t_{m}$. By $\tilde{\mathbf{w}}_{i}^{m}$ we shall denote approximate solution of $\tilde{\mathbf{w}}$ on the element $K_{i}$ at time instant $t_{m}$

$$
\tilde{\mathbf{w}}_{i}^{m} \approx \frac{1}{\left|K_{i}\right|} \int_{K_{i}} \tilde{\mathbf{w}}\left(x, t_{m}\right) \mathrm{d} x
$$

For the numerical flux $\tilde{\mathbf{H}}$ we again use the concept of the Vijayasundaram numerical flux in the following way

$\tilde{\mathbf{H}}\left(\tilde{\mathbf{w}}_{i}^{m}, \tilde{\mathbf{w}}_{j}^{m}, \boldsymbol{n}_{i j}\right)=\left(\langle\mathbf{v}\rangle \cdot \boldsymbol{n}_{i j}\right)^{+} \tilde{\mathbf{w}}_{i}^{m}+\left(\langle\mathbf{v}\rangle \cdot \boldsymbol{n}_{i j}\right)^{-} \tilde{\mathbf{w}}_{j}^{m} \quad j \in s(i)$, $\tilde{\mathbf{H}}\left(\tilde{\mathbf{w}}_{i}^{m}, \tilde{\mathbf{w}}_{j}^{m}, \boldsymbol{n}_{i j}\right)=\left(\mathbf{v} \cdot \boldsymbol{n}_{i j}\right)^{+} \tilde{\mathbf{w}}_{i}^{m}+\left(\mathbf{v} \cdot \boldsymbol{n}_{i j}\right)^{-} \tilde{\mathbf{w}}_{B} \quad j \in \gamma(i)$,

where $\tilde{\mathbf{w}}_{B}$ denotes boundary conditions. One possibility of linearization of $\tilde{\mathbf{R}}_{s}$ and $\tilde{\mathbf{s}}$ is via Taylor expansion which is used in our case. For more details see [6]. 


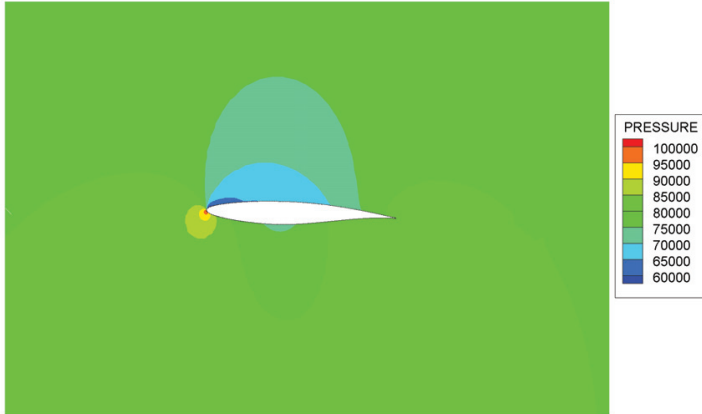

Figure 1. The pressure distribution for the case 3 .

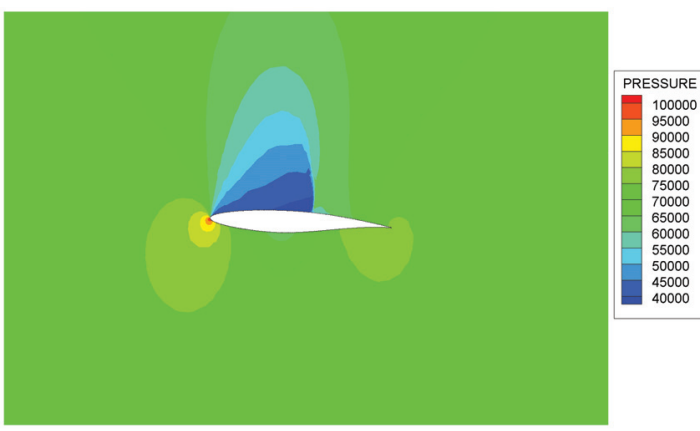

Figure 2. The pressure distribution for the case 9 .

\section{Numerical experiments}

In order to demonstrate the applicability of the developed method we shall present two numerical simulations of compressible turbulent flow in 2D. For this purpose we chose two regimes for the profile RAE2822 and compared it with experimental data, which can be found in [1]. First regime is characterized by the far-field Mach number $M_{\infty}=0.6$ and the angle of the attack $\alpha=2.57^{\circ}$ ('case 3' in [1]) and second case is characterized by the Mach number $M_{\infty}=$ 0.73 and the angle of the attack $\alpha=3.19^{\circ}$ ('case 9' in [1]). Figures 1 and 2 show the pressure distribution. Comparison of the computed pressure coefficient $c_{p}$ with the experimental data is shown in Figures 3 and 4.

\section{Conclusion}

In this paper we dealt with the finite-volume - space-time discontinuous Galerkin method for the numerical solution of compressible turbulent flow. The applicability of the proposed method was demonstrated on the examples, which show, that the presented method is an efficient numerical scheme for the solution of the $2 \mathrm{D}$ compressible turbulent flow.

\section{Acknowledgment}

This result originated with the support of Ministry of Industry and Trade of the Czech Republic for the long-term

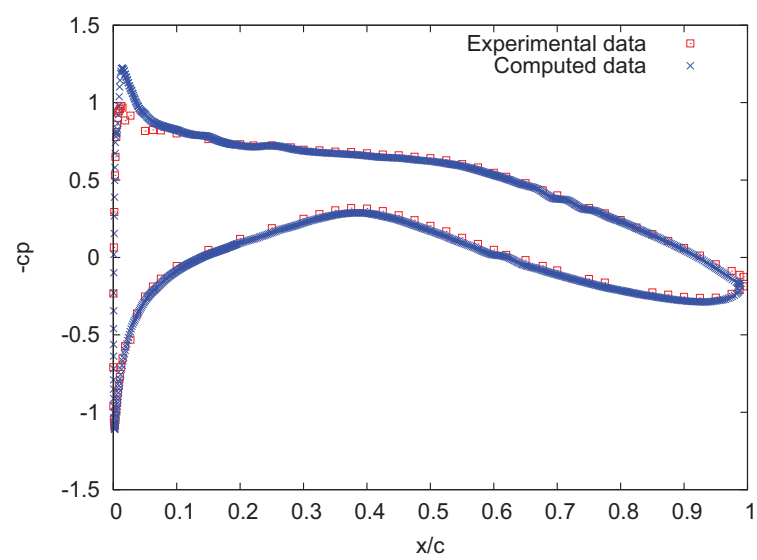

Figure 3. Comparison of the computed pressure coefficient $c_{p}$ with the experimental data for the case 3 .

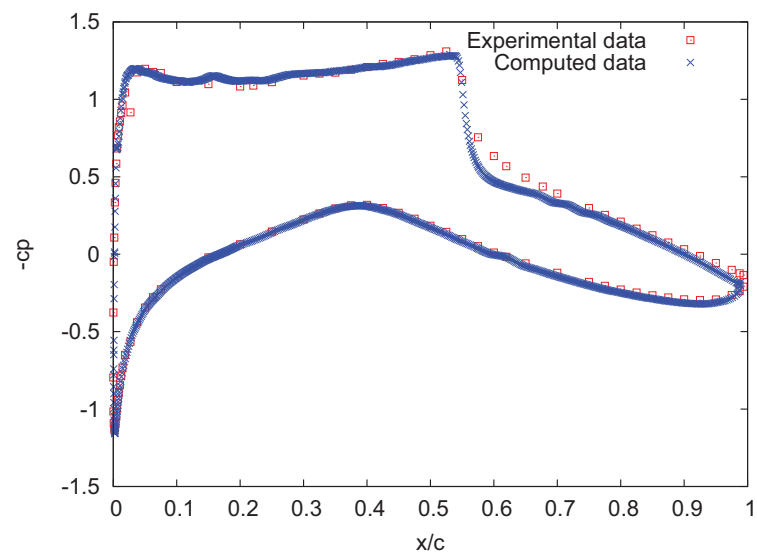

Figure 4. Comparison of the computed pressure coefficient $c_{p}$ with the experimental data for the case 9 .

strategic development of the research organisation. The authors acknowledge this support.

\section{References}

1. Cook P. H., McDonald M. A. and Firmin M. C. P. AGARD Advisory Report 138, (1979)

2. Česenek J. Discontinuous Galerkin method for solving compressible viscous flow. Doctoral Thesis, (2011)

3. Česenek J. et al., Appl. Math. Comput., doi:10.1016/1.amc.2011.08.077 (2011)

4. Česenek J., Feistauer M., Kosik A. ZAMM - Z. Angew. Math. Mech. 93, 6 - 7, 387 - 402 (2013)

5. Česenek J. Experimental fluid mechanics,142-147 (2013)

6. Česenek J. Combined finite volume - space-time discontinuous Galerkin method for the 2D compressible turbulent flow. Technical report R-6154, VZLÚ, Beranových 130, Prague (2014)

7. Dolejší V., Feistauer M. Discontinuous Galerkin Method. Springer, Heidelberg (2015)

8. Feistauer M., Felcman J., and Straškraba I. Mathematical and Computational Methods for Compressible Flow. Clarendon Press, Oxford (2003)

9. Kok C.J. AIAA Journal, Vol. 38. No. 7., (2000)

10. Sváček P., Feistauer M., Horáček J. J. Fluids Struc., 23, 391-411 (2007) 